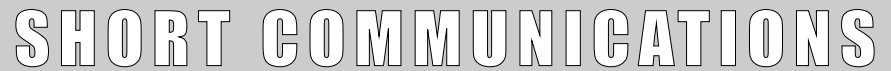

\section{QTF-Gold assay for monitoring of anti-tuberculosis therapy in subjects with active TB}

\author{
Ilaria Sauzullo', Fabio Mengoni', Raffaella Rossi', Miriam Lichtner', Maria Cecilia Rizza', \\ Fernanda Chiarini' ${ }^{2}$, Claudio Maria Mastroianni ${ }^{3}$, Vincenzo Vullo' $^{\prime}$
}

'Dipartimento di Malattie Infettive e Tropicali, “ Sapienza” Università di Roma

Dipartimento di Scienze di Sanità Pubblica, " Sapienza” Università di Roma

Dipartimento di Malattie Infettive e Tropicali, “ Sapienza” Università di Roma, Polo Pontino

Key words: M. tuberculosis, QFT-Gold,TB-therapy, eradication.

\section{QuantiFERON-TB Gold nel monitoraggio terapeutico dell'infezione tubercolare}

\section{SUMMARY}

Introduction: The identification and characterization of two M. tuberculosis-specific antigens (ESAT-6 and CFP10) has led to the development of a whole blood new generation of $M$. tuberculosis specific diagnostic tests, that have several advantages over tuberculin skin test (TST), in terms of higher specificity, better correlation with surrogate measures of exposure to $M$. tuberculosis in low-incidence setting, and less cross-reactivity with $M$. bovis (BCG) vaccine and environmental mycobacteria. The role of these new tests in evaluating post-therapy tuberculosis eradication has not been investigated yet. Aim of this longitudinal study was to determinate changes of response to $M$. tuberculosis-specific antigens in patients during the standard tuberculosis treatment and to investigate the in vitro effects of tuberculosis drugs on the IFN- $\gamma$ release.

Methods: 23 individuals with active tuberculosis were enrolled and followed over time. They were tested with QuantiFERON TB-Gold (QFT-Gold) at four time points: at diagnosis ( $\mathrm{t} 0$ ), after 3 and 6 months of treatment ( $\mathrm{tl}$ t2) and at the end of the specific treatment ( $\mathrm{t} 3)$.

Results: At baseline all patients were positive by QFT-Gold. At second time-point 17 out of $23(74 \%)$ were positive, at third time-point II of $23(47 \%)$ were positive, at treatment completion 3/23 (13\%) were positive. The conversion to negative response to M. tuberculosis-specific antigens was found in $87 \%$ patients analyzed after successful therapy. Longitudinal QFT-Gold testing shown a significant decrease $(p<0.00 \mathrm{I})$ of IFN- $\gamma$ response during the therapy.

No inhibitory effect on IFN- $\gamma$ release was noted in vitro for chemotherapy using at concentrations compatible with those achieved in serum of treated patients. We have revealed an inhibitory effect only at concentrations 2-3 times greater than those previously tested.

Conclusion: A successful therapy for tuberculosis causes a significant decrease of the ESAT-6 and CFP-10 response and this data suggest that the QTF-Gold assay may have a role as a tool for evaluating tuberculosis eradication after standard tuberculosis treatment.

\section{INTRODUZIONE}

Il tasso di incidenza della tubercolosi (TB), così come riportato dall'Organizzazione Mondiale della Sanità, è in aumento in tutto il mondo e tale patologia rappresenta, quindi, tra le malattie infettive, la più importante causa di morte dopo l'infezione da HIV $(3,8)$. Sebbene il trattamento farmacologico degli individui con tubercolosi attiva rappresenti la misura più importante nell'intervento di controllo dell'infezione, nei Paesi a bassa incidenza sono altrettanto rilevanti il riconoscimento, la sorveglianza e il trattamento preventivo delle persone affette da infezione latente e ad alto rischio di sviluppare la malattia.

Il trattamento specifico antitubercolare mira non solo al controllo della fase attiva della malattia, ma soprattutto all'eradicazione per impedire le recidive/riattivazioni della malattia. Purtroppo non esistono ad oggi metodiche per valutare l'avvenuta eradicazione dell'infezione tubercolare.

La diagnosi di malattia attiva, posta sulla base del sospetto clinico-radiologico e/o della presenza di uno stato di infezione tubercolare, è confermata dalla dimostrazione della presenza di M. tubercu-

\section{Corresponding author: Sauzullo llaria}

Dipartimento di Malattie Infettive e Tropicali, Università La Sapienza di Roma, - Policlinico Umberto I, 00 I6I Roma Tel.: 06-4997088। Fax:06-49972625 - E-mail: ilariasauzullo@libero.it 
losis. La diagnosi di infezione tubercolare latente, invece si basa sulla dimostrazione di una risposta immunitaria ad antigeni micobatterici, in assenza di segni di malattia in atto.

Tale risposta immunitaria, fino al 2001, poteva essere misurata solo attraverso la reazione cutanea all'iniezione intradermica di tubercolina (7), ma attualmente può essere individuata anche attraverso la misurazione della produzione di interferone gamma da parte dei linfociti $T$ stimolati in vitro (test IFN- $\gamma$ ). La misurazione di una sensibilizzazione contro gli antigeni tubercolari è prova indiretta della presenza di un'infezione tubercolare latente $o$ attiva (1), ma né i test alla tubercolina né i test IFN- $\gamma$ permettono di distinguere tra un'infezione tubercolare latente e una tubercolosi attiva (9).

Recenti studi di analisi genomica comparativa hanno consentito l'identificazione di proteine secretorie specifiche di M. tuberculosis, denominate ESAT-6 (early secretory antigen target-6) e CFP-10 (culture filtrate protein-10), codificate dalla regione genomica RD1 (region of differentiation-1), presenti in modo peculiare in M. tuberculosis ed assenti, invece, in tutti i ceppi di $M$. bovis (BCG) e nella maggior parte dei micobatteri non tubercolari (NTM), esclusi $M$. kansassii, $M$. szulgai, M. marinum $(2,6,15)$. L'utilizzo di queste due proteine come stimolo antigenico ha portato allo sviluppo di nuovi test per la diagnosi immunologica dell'infezione tubercolare dotati di elevata sensibilità e specificità.

Questi test, denominati con l'acronimo di IGRA (interferon gamma releasing assays), si basano sul principio che i linfociti $\mathrm{T}$ effettori di individui sensibilizzati con antigeni specifici, producono l'IFN- $\gamma$ quando vengono nuovamente in contatto con gli stessi antigeni. Si presume, perciò, che un'elevata produzione di IFN- $\gamma$ possa essere indicativa di infezione tubercolare $(13,14)$.

I due test immunologici attualmente disponibili in commercio, evoluzione di precedenti tipologie di kit, sono il QuantiFERON-TB Gold (Cellestis Ltd, Carnegie, Victoria, Australia) a cui si aggiunge la successiva versione "In Tube", e il TSPOT.TB (Oxford Immunotec, Abingdon, Gran Bretagna). I due test sono simili, in quanto entrambi misurano l'immunità cellulo-mediata valutando quantitativamente l'IFN- $\gamma$ secreto dai linfociti $\mathrm{T}$ in risposta ad antigeni tubercolari, ma presentano alcune differenze metodologiche.

Il QuantiFERON-TB Gold viene eseguito direttamente su campione di sangue venoso intero e la stimolazione antigenica, che si basa sull'utilizzo di peptidi di ESAT-6 e CFP-10, avviene a $37^{\circ} \mathrm{C}$ per 16-24 ore. Al termine del periodo di incubazione, i campioni di plasma possono venire conservati a $2-8^{\circ} \mathrm{C}$ fino a 8 settimane e la determina- zione della produzione di IFN- $\gamma$ secreto è effettuata mediante tecnica "Enzime Linked Immuno Assay" (ELISA). Il test T-SPOT.TB, viene eseguito usando cellule mononucleate purificate, isolate da un campione di sangue venoso periferico. La stimolazione antigenica viene eseguita su piastra mediante peptidi di ESAT-6 e CFP-10 cui un numero prefissato di cellule viene esposto per 1624 ore, in pozzetti separati per ciascun antigene, a $37^{\circ} \mathrm{C}$ in un atmosfera controllata di anidride carbonica al $5 \%$.

La produzione di IFN- $\gamma$ viene analizzata mediante tecnologia "Enzyme-linked immunospot" (ELISPOT), che consente di rilevare ogni singolo linfocita che produce la citochina sotto forma di spot o impronta lasciata dall'IFN- $\gamma$ prodotto dalle cellule e legato dagli anticorpi anti-IFN- $\gamma$ che rivestono la piastra stessa. La quantificazione del risultato viene effettuata mediante conta del numero di spot con un apposito lettore di piastre. Il QuantiFERON-TB Gold è stato approvato nel maggio 2001 dalla Food and Drug Administration (FDA) e inserito, come alternativa al test cutaneo per la TB latente, nelle linee guida del Center for Disease Control and Prevention di Atlanta (10, 11).Entrambi i test (QuantiFERON-TB Gold e TSPOT.TB) sono stati approvati dalle linee Guida Britanniche stilate dal National Institute for Health and Clinical Excellence (12) che raccomandano una strategia a due livelli in cui la Mantoux rimane il test di screening che, se positivo o inattendibile, dovrebbe essere confermato da uno dei test IGRA. Tale protocollo è tuttora in discussione e necessita di una valutazione su larga scala per l'impatto sia clinico che economico. Simile proposta compare nelle linee guida congiunte delle Società Italiane di Pneumologia (AIPO-SIMER) (5).

L'obiettivo dello studio era quello di valutare l'utilizzo clinico del QuantiFERON-TB Gold (QFT-Gold) come parametro per il monitoraggio della terapia antitubercolare e studiare l'effetto immunomodulante dei chemioterapici sul rilascio di IFN- $\gamma$ da parte dei linfociti T.

\section{MATERIALI E METODI Popolazione in studio}

È stato condotto uno studio longitudinale su 23 pazienti adulti con diagnosi di tubercolosi attiva (70\% TB polmonare e 30\% TB extrapolmonare), seguiti presso il Dipartimento di Malattie Infettive del Policlinico Umberto I "Sapienza" di Roma. Dei 23 pazienti il 58\% erano uomini e il $42 \%$ donne, il $46 \%$ dei soggetti non era di nazionalità italiana e nessuno presentava una condizione di immunodeficienza.

La diagnosi di tubercolosi è stata formulata con 
identificazione di M. tuberculosis mediante metodi microbiologici/istologici e/o con criteri clinicodiagnostico strumentali ( $\mathrm{Rx}, \mathrm{TAC})$ associati ad una valida risposta alla terapia antitubercolare.

Tutti i soggetti sono stati sottoposti ad una terapia antitubercolare specifica con 4 farmaci e sono stati valutati con il test QFT-Gold a quattro tempi: alla diagnosi ( $\mathrm{t} 0)$, dopo 3 e 6 mesi di terapia ( $\mathrm{t} 1 \mathrm{e}$ t2) e a fine trattamento terapeutico ( $\mathrm{t} 3=9$ mesi).

I risultati ottenuti con il QFT-Gold sono stati confrontati con i dati clinici raccolti durante l'anamnesi e con gli esiti degli esami microbiologici durante il follow-up.

\section{Test QuantiFERON® TB-Gold}

Il QuantiFERON-TB Gold è un test diagnostico (Cellestis Ltd, Carnegie, Victoria, Australia) eseguito ex vivo su un campione di sangue periferico che permette la valutazione della risposta immunitaria cellulo-mediata ad antigeni specifici per $M$. tuberculosis: ESAT-6 (early secretory antigen target-6) e CFP-10 (culture filtrate protein-10).

Il test si svolge in due fasi:

Nella prima fase si procede all'incubazione di quattro aliquote da $1 \mathrm{ml}$ di sangue intero eparinato rispettivamente con gli antigeni specifici ESAT6 e CFP-10, il mitogeno (phytoaemagglutinin) come controllo positivo e il Nil come controllo negativo.

Nella seconda fase, dopo l'incubazione di $12-18 \mathrm{~h}$ a $37^{\circ} \mathrm{C}$, si preleva il plasma e si quantifica IFN- $\gamma$ prodotto mediante saggio ELISA. Una curva con IFN- $\gamma$ ricombinata è effettuata ad ogni determinazione. I risultati dei campioni sono espressi in Unità Internazionali $/ \mathrm{ml}(\mathrm{UI} / \mathrm{ml})$ riferite a questa preparazione standard. Il soggetto è ritenuto positivo per l'infezione da $M$. tuberculosis se la produzione di IFN- $\gamma$ verso gli antigeni ESAT-6 e/o CFP10 supera il cut-off stabilito di $0.35 \mathrm{UI} / \mathrm{ml}$. L'analisi di dati è stata fatta utilizzando il QuantiFERON®-TB Analisi Software.

\section{Effetto in vitro dei farmaci antitubercolari sul} rilascio di IFN- $\gamma$

Per valutare l'interferenza dei farmaci antitubercolari sulla produzione ex-vivo di IFN- $\gamma$ sono state allestite una serie di prove in cui aliquote di $0.5 \mathrm{ml}$ di sangue eparinato sono state stimolate con il mitogeno utilizzato nel QFT-Gold, (PHA, phytoaemagglutinin) e coincubate per $18 \mathrm{~h}$ a $37^{\circ} \mathrm{C}$ con i seguenti farmaci: Streptomicina, Isoniazide, Rifampicina ed Etambutolo. I test sono stati allestiti con tre diverse concentrazioni a raddoppio dei farmaci partendo dalle rispettive CMax raggiunte nel plasma dei soggetti in trattamento (INH $5 \mathrm{mg} / \mathrm{ml}$, RIF $7 \mathrm{mg} / \mathrm{ml}$, ETB $5 \mathrm{mg} / \mathrm{ml}, \quad$ STR $40 \mathrm{mg} / \mathrm{ml}$ ). Il rilascio di IFN- $\gamma$ è stato quantificato mediante saggio ELISA standard (QuantiFERONCMI, Cellestis Ltd, Carnegie, Victoria, Australia).
La vitalità cellulare è stata valutata mediante colorazione con trypan blue.

\section{Analisi statistica}

La produzione di IFN- $\gamma$ in risposta a stimolazione antigenica è stata espressa in Unità Internazionali $(\mathrm{IU} / \mathrm{mL})$. Per i diversi parametri analizzati è stata calcolata la Media ( \pm SE) e la Mediana (range). I dati, nei vari tempi, sono stati comparati utilizzando il test non parametrico di Wilcoxon sui ranghi. Per gli esperimenti in vitro è stato effettuato il ttest. Una $\mathrm{p}<0.05$ è stata valutata come significativa.

\section{RISULTATI}

\section{Risultati dell'analisi longitudinale nei pazienti in trattamento}

È stato condotto uno studio longitudinale su 23 pazienti con diagnosi di tubercolosi attiva, confermata a livello clinico-microbiologico. I soggetti sono stati seguiti per 9 mesi e analizzati con il test QFT-Gold eseguendo prelievi ematici prima dell'inizio della terapia (t0), durante ( $\mathrm{t} 1: 3$ mesi; $\mathrm{t} 2: 6$ mesi) e a fine trattamento (t3: 9 mesi).

Alla diagnosi tutti i pazienti mostrano un risultato positivo al QFT-Gold (t0: Mediana IFN- $\gamma 4.61$ $\mathrm{UI} / \mathrm{ml}$, range $0.35-29.29$ ). I risultati del test ai vari tempi sono stati i seguenti: al t1 erano positivi 17/23 pazienti ( $\mathrm{t} 1: 1.03 \mathrm{UI} / \mathrm{ml}$, range $0-27.67$ ), al t2 $11 / 23$ (t2: $0.305 \mathrm{UI} / \mathrm{ml}$, range $0-9)$, al t3 $3 / 23$ (t3: $0.05 \mathrm{UI} / \mathrm{ml}$, range $0-7$ ). A fine trattamento terapeutico, quindi, nel $87 \%$ dei pazienti si evidenziava una conversione negativa del test QFTGold rispetto all'arruolamento.

Complessivamente $\mathrm{i}$ test effettuati longitudinalmente hanno evidenziato un progressivo decremento significativo $(\mathrm{p}<0.001)$ della produzione di IFN- $\gamma$ durante la terapia (figura I).

\section{Risultati dell'effetto in vitro dei farmaci antitu- bercolari sul rilascio di IFN- $\gamma$}

Gli esperimenti in vitro, effettuati per valutare l'effetto immunomodulante dei farmaci antitubercolari, hanno mostrato come questi, utilizzati alle loro concentrazioni plasmatiche $(\mathrm{C} \operatorname{Max}=\mathrm{C} 1)$, non influenzino il rilascio di IFN- $\gamma$ da parte delle cellule T rispetto al controllo (CNT). È stato invece osservato che, dosi maggiori di farmaci $(\mathrm{C} 2$, C3) presentano un effetto inibitorio statisticamente significativo $(\mathrm{p}<0.05)$. Si evidenzia, dunque, una down regolazione dose-dipendente sulla produzione di IFN- $\gamma$ (figura II).

Dopo aver osservato l'effetto inibitorio indotto dai farmaci ad alte concentrazioni, ne abbiamo valutato il loro eventuale effetto tossico sulla sopravvivenza cellulare.

I risultati mostrano assenza di tossicità farmacologica come dimostrato dall'alta \% di sopravvivenza cellulare $(87 \%)$ (figura III). 


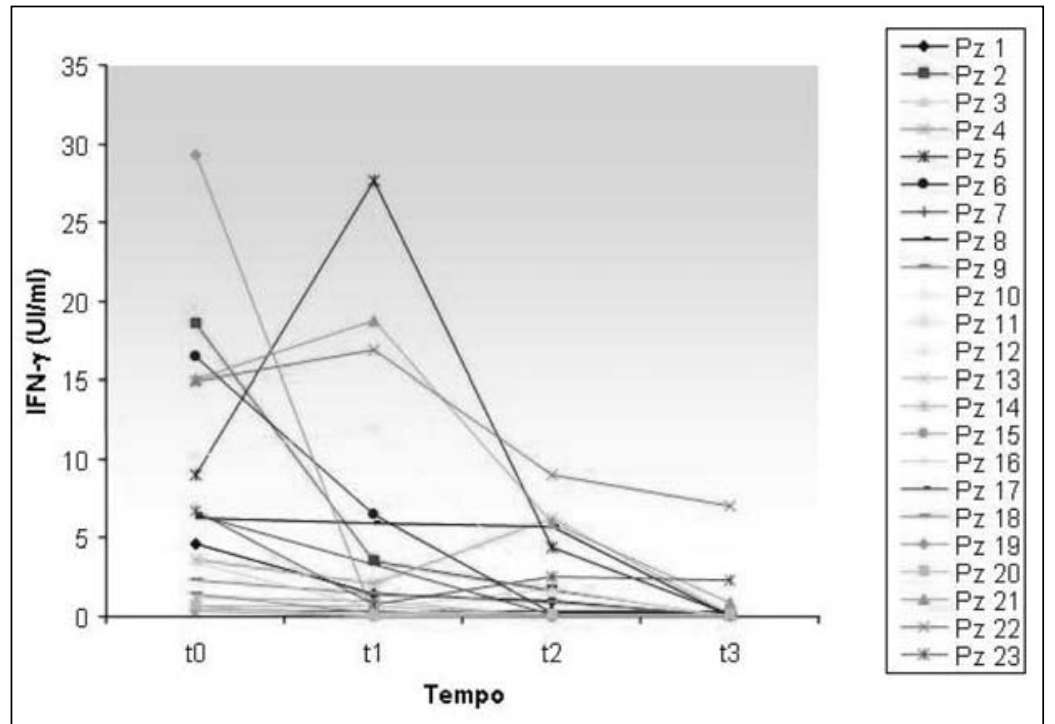

Figura I. Analisi longitudinale nei 23 pazienti con TB attiva.

Produzione di IFN- $\gamma(\mathrm{Ul} / \mathrm{ml})$ in risposta agli antigeni tubercolari prima dell'inizio della terapia ( $\mathrm{t} 0)$, durante $(\mathrm{tl}: 3$ mesi; $\mathrm{t} 2: 6$ mesi) e a fine trattamento terapeutico (t3: 9 mesi). Si osserva un decremento significativo $(p<0.00 \mathrm{I})$ del rilascio di IFN- $\gamma$.

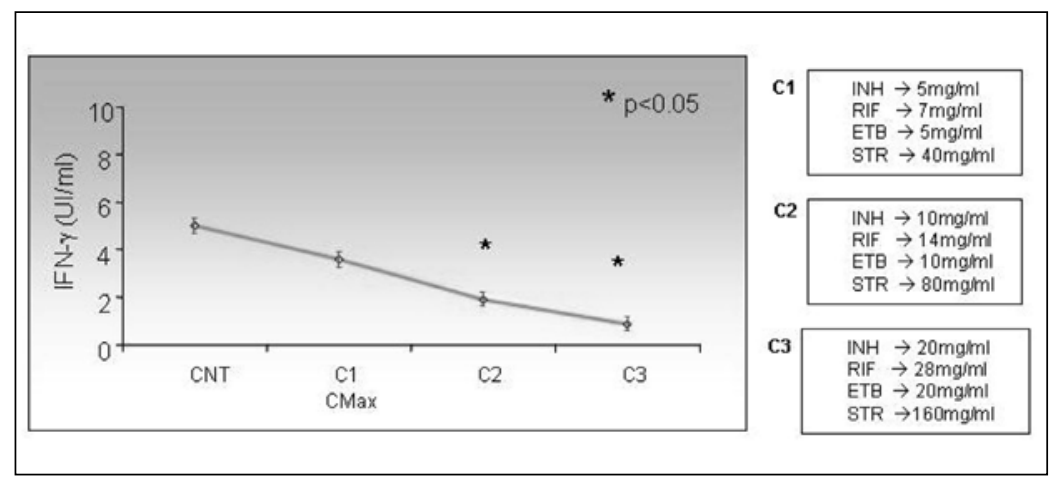

Figura II. Influenza dei farmaci antitubercolari sul rilascio di IFN- $\gamma$.

I 4 farmaci antitubercolari alle loro concentrazioni plasmatiche $(\mathrm{C} \mathrm{Max}=\mathrm{Cl})$ non influenzano il rilascio di IFN- $\gamma$ da parte delle cellule T rispetto al controllo (CNT). Concentrazioni maggiori di farmaci $(\mathrm{C} 2, \mathrm{C} 3)$ presentano un effetto inibitorio. Tale differenza è statisticamente significativa $(p<0.05)$.

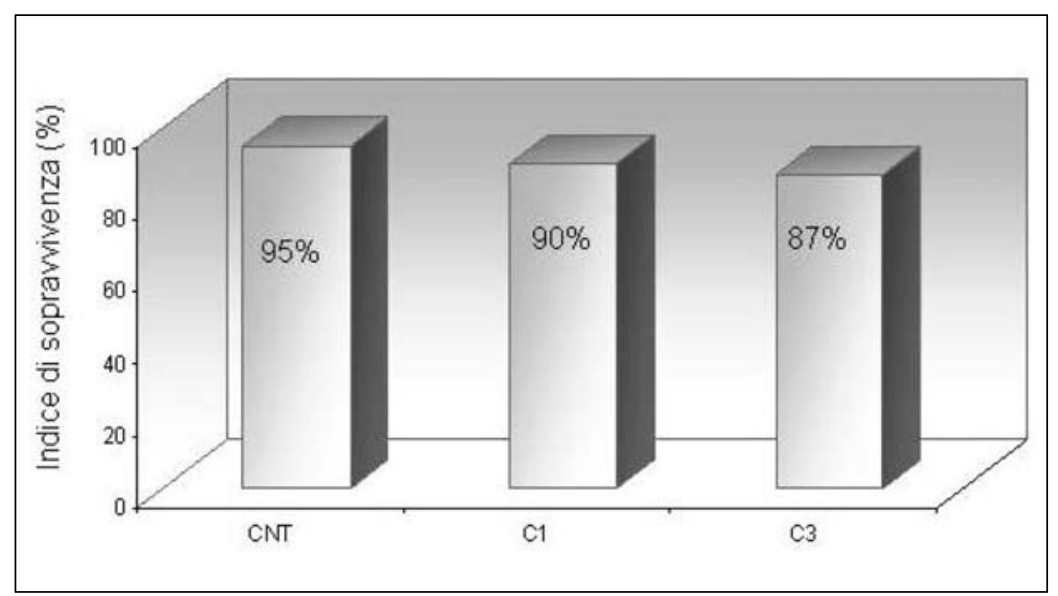

Figura III. Influenza dei farmaci antitubercolari sulla sopravivenza cellulare I farmaci antitubercolari utilizzati ad alta concentrazione (C3) non mostrano effetto tossico sulla sopravvivenza cellulare.

\section{DISCUSSIONE}

Il trattamento dei casi di tubercolosi attraverso schemi terapeutici standardizzati a più farmaci consente di aumentare il tasso di successo terapeutico della malattia e di limitare l'insorgenza delle forme, particolarmente temibili, resistenti ai farmaci antitubercolari di prima scelta.

L'obiettivo della terapia è l'eradicazione della malattia tubercolare con conseguente estinzione delle possibili riattivazioni. Nessun test, purtroppo, consente oggi di valutare tale eradicazione, in quanto la malattia potrebbe rimanere in uno stato di latenza senza dare segni clinico-radiologici e microbiologici.

Per valutare il possibile utilizzo del QuantiFERON-TB GOLD, come parametro per il monitoraggio terapeutico dell'infezione tubercolare e relativa eradicazione, è stato effettuato uno studio longitudinale su un gruppo di 23 pazienti con TB attiva in corso di trattamento specifico.

I pazienti sono stati seguiti per 9 mesi con valutazione del test prima, durante e a fine trattamento.

L'analisi dei test effettuati longitudinalmente evidenzia un progressivo decremento significativo $(p<0.001)$ della produzione di IFN- $\gamma$, in risposta a stimoli specifici, durante la terapia. Nell' $87 \%$ dei pazienti si evidenzia una conversione negativa del test QFTGold rispetto a quanto evidenziato al momento della diagnosi, risultando tali pazienti in fase di guarigione anche dal punto di vista clinico, microbiologico e strumentale.

La negativizzazione del test, a fine trattamento terapeutico, suggerisce una possibile claerance del micobatterio e, diversamente dal TST, che rimane positivo dopo il trattamento, il QFT-Gold può fornire ulteriori informazioni sulla eradicazione della patologia. Infatti la produzione di IFN$\gamma$, rilevata dal test, sarebbe dovu- 
ta non tanto ad una risposta da parte dei linfociti $\mathrm{T}$ memory, ma da parte dei linfociti $\mathrm{T}$ effettori, attivati da una persistenza degli antigeni nell'organismo.

Numerose evidenze sperimentali e cliniche testimoniano come diversi antibiotici, oltre all'attività antibatterica, esercitino importanti interazioni sul sistema immunitario dell'organismo, è quindi importante conoscere sia la natura che la portata di eventuale loro influenza sulla risposta immunitaria $(4,16)$.

Tuttavia, in corso di terapia specifica, un esito negativo del test immunologico in vitro potrebbe invece essere influenzato da un effetto inibitorio dei farmaci sulla produzione di IFN- $\gamma$, ragion per cui, abbiamo condotto alcuni esperimenti in vitro che dimostrano, contrariamente, come i farmaci antitubercolari, alle comuni concentrazioni plasmatiche, non inibiscano il rilascio di IFN- $\gamma$. Dosi maggiori hanno, invece, un effetto inibitorio, risultato che conferma $\mathrm{i}$ dati già presenti in letteratura sul possibile effetto immunomodulante di altri antibiotici $(4,16)$.

La negativizzazione del test, a fine trattamento terapeutico, unito ad un outcome clinico favorevole, evidenzia un possibile utilizzo del test ai fini del monitoraggio terapeutico valutando la possibile interferenza dei farmaci sul rilascio di IFN- $\gamma$ ad alte concentrazioni plasmatiche.

È ovvio che l'osservazione clinica, radiologica e le indagini microbiologiche rappresentano ancora oggi il gold standard per valutare l'andamento della malattia tubercolare. Un follow-up a lungo termine sarà necessario per stabilire se la negativizzazione del test QFT-Gold, a fine trattamento specifico, può essere indicativa di eradicazione dell'infezione tubercolare.

\section{BIBLIOGRAFIA}

1. Andersen P, Munk ME, Pollock JM. Specific immunebased diagnosis of tuberculosis. Lancet 2000; 356: 1099-104.
2. Berthet FX, Rasmussen PB, Rosenkrands I, et al. $M$. tuberculosis operon encoding ESAT- 6 and a novel low-molecular-mass culture filtrated protein CFP-10. Microbiology 1998; 144: 3195-203.

3. Bloom BR. Tuberculosis, the global view. $N$ Engl $J$ Med 2002; 346(19): 1434-5.

4. Daschner FD. Antibiotics and host defence with special reference to phagocytosis by human polymorphonuclear. J Antimicrob Chemoter 1985; 16: 135-41.

5. Documento sull'utilizzo dei nuovi test immunologici per la diagnosi di infezione tubercolare latente. Rass Patol App Respir 2007; 22: 13-20.

6. Harboe M, Oettinger T, Wiker HG, et al. Evidence for occurrence of the ESAT-6 protein in M. tuberculosis and virulent $M$. bovis and for its absence in $M$. bovis BCG. Infect Immun 1996; 64: 116-22.

7. Huebner RE, Schein MF, Bass JB. The tuberculin skin test. Clin Infect Dis 1993; 17: 968-75.

8. Kapp C. Global fund on AIDS, tuberculosis, and malaria holds first board meeting. Lancet 2002; 359(9304): 414.

9. Lega polmonare svizzera: Manuale della tubercolosi. Maggio 2007, Dipartimento federale dell'interno (DFI), Ufficio federale della sanità pubblica (UFSP).

10. Mazurek GH, Jereb JJ, LoBue P, Iademarco MF, Metchock B, Vernon A. Guidelines for Using the QuantiFERON®-TB Gold Test for Detecting Mycobacterium tuberculosis Infection, United States MMWR RR 2005; 54(RR15): 49-55.

11. Mazurek GH, Villarino ME. Guidelines for using the QuantiFERON-TB test for diagnosing latent $M$. tuberculosis infection. Center for Disease Control and Prevention. MMWR Recomm Rep 2003; 52(RR-2): 15-8.

12. National Institute for Health and Clinical Excellance. Clinical Guideline 33 Tuberculosis: clinical diagnosis and management of tuberculosis, and measures for its prevention and control. March 2006.

13. Pai M, Kalantri S, Dheda K. New tools and emerging technologies for the diagnosis of tuberculosis: Part 1. Latent tuberculosis. Expert Rev Mol Diag 2006; 6(3): 423-32.

14. Pai M, Lee WR, Colford JM. Interferon- assays in the immunodiagnosis of tuberculosis: a systematic review. Lancet Infect Dis 2004; 4: 761-76.

15. Sorensen AL, Nagai S, Houen G, et al. Purification and characterization of a low-molecular-mass T-cell antigen secreted by $M$. tuberculosis. Infect Immun 1995; 63: 1710-7.

16. Welch WD, et al. Effect of antimicrobial agents on human polymorphonuclear leucocyte microbicidalfuction. Antimicrob. Agents Chemoter 1981; 20: 15-20. 